\title{
Generation and characterization of a polyclonal antibody against human high mobility group box 4
}

\author{
FEN YANG ${ }^{1,2}$, RUNSHENG LI $^{2}$, AIZHEN HONG $^{2}$, FEI DUAN $^{1,2}$ and YUHUA LI ${ }^{2}$ \\ ${ }^{1}$ Shanghai Medical College, Fudan University; ${ }^{2}$ Key Laboratory of Contraceptive Drugs and Devices of NPFPC, \\ Shanghai Institute of Planned Parenthood Research, Shanghai 200032, P.R. China
}

Received March 4, 2013; Accepted September 3, 2013

DOI: $10.3892 / \mathrm{mmr} .2013 .1701$

\begin{abstract}
A human high mobility group box 4 (hHMGB4) expression construct (pET-28a/hHMGB4) was generated by cloning the hHMGB4 full-length cDNA in the expression vector $\mathrm{pET}-28 \mathrm{a}(+)$. The hHMGB4 fusion protein with $\mathrm{His}_{6}$-Tag was prepared using E.coli BL21 (DE3) transformed with pET-28a/hHMGB4 and purified via preparative SDS-PAGE plus electroelution. Immunization of rabbits with the purified hHMGB4 generated polyclonal antibodies. The titer of the antiserum was determined to be 1:102,400 by ELISA analysis. Western blotting analysis showed that the antibody specifically recognized the recombinant hHMGB4 protein and also the endogenous hHMGB4 protein in prostate cancer cells. In addition, immunohistochemical staining analysis using the prepared antibody revealed marked hHMGB4 staining in the nuclei of the human prostate tissue. These data demonstrate that the anti-hHMGB4 polyclonal antibody may be a useful reagent for the functional study of hHMGB4.
\end{abstract}

\section{Introduction}

The high mobility group box (HMGB) gene family, is the most abundant type of non-histone chromatin binding protein in eukaryotes and is comprised of four members: HMGB1, HMGB2, HMGB3 and HMGB4 (1). Mammalian HMGBs are characterized by two highly conserved and tandem DNA binding domains, HMG boxes $\mathrm{A}$ and $\mathrm{B}$, followed by a long acidic C-terminal tail (2). Proteins in this family may bind noncanonical DNA structures, including single-stranded DNA with their own HMG-box and act as a chromatin chaperone through distorting DNA (3). HMGBs also take part in nucleosome-remodeling, enhanceosome organization and tran-

Correspondence to: Mrs. Yuhua Li, Key Laboratory of Contraceptive Drugs and Devices of NPFPC, Shanghai Institute of Planned Parenthood Research, 2140 Xietu Road, Shanghai 200032, P.R. China

E-mail: 1i_yuhua@163.com

Key words: E. coli BL21 (DE3), human high mobility group box 4, pET-28a(+), polyclonal antibody scription regulation by interacting directly with nucleosomes and enhancesomes, as well as transcription factors $(1,4)$. To date, three members of the HMGB family, including HMGB1, HMGB2 and HMGB3, have been studied. In adult vertebrates, HMGB1 is ubiquitously expressed in all cell types and its expression level is found to be correlated with the differentiation stage of cells (5). HMGB2 is abundant in the thymus, lymphoid organs and testes in adult mice and it is also found in all human and mouse immortalized cell lines $(5,6)$, whereas HMGB3 is primarily present in primitive hematopoietic cells (7). HMGB4, as a novel member of the HMGB family, also contains two highly conserved and tandem DNA binding domains, HMG boxes $\mathrm{A}$ and $\mathrm{B}$, however lacks the acidic C-terminal tail, which exists in all other HMGB proteins (8). As a transcriptional repressor, HMGB4 is highly expressed in adult mouse testis, lowly in brain and not expressed in other tissues (8). However, the distribution and function of HMGB4 in various human tissue remains unknown. In the present study, the development of a specific anti-hHMGB4 polyclonal antibody was reported. Moreover, the characteristics of the prepared antibody were evaluated by ELISA, western blotting and immunohistochemical techniques.

\section{Materials and methods}

Construction of the recombinant expression plasmid. The hHMGB4 fragment was amplified from the plasmid pBluescriptR/hHMGB4 harboring full-length hHMGB4 cDNA (purchased from Gene Copoeia, Rockville, MD, USA) by PCR. Gene-specific primer pairs were designed as follows: forward, 5'-GCGCGAATTCATGGGAAAAGAAATCCAG-3' (the EcoRI recognition site underlined); reverse, 5'-CTAGTCGAC GCTCTGCCTGACTCTTTTCCC-3' (the SalI recognition site underlined). The amplified PCR product was purified, digested with EcoRI and SalI and ligated to the pET28a(+) vector (Novagen, Darmstadt, Germany), which was digested by the same restriction enzymes to produce the expression plasmid pET28a(+)/hHMGB4. Next, the expression plasmid was transformed into E.coli $\mathrm{DH} 5 \alpha$-competent cells and plated on Luria Broth (LB) plates containing $50 \mu \mathrm{g}$ kanamycin $/ \mathrm{ml}$. Ten single colonies of E.coli DH5 $\alpha$-pET28a(+)/HMGB4 cells were inoculated into the LB medium, including $50 \mu \mathrm{g}$ kanamycin $/ \mathrm{ml}$ at $37^{\circ} \mathrm{C}$ overnight. The plasmid DNA was extracted using Plasmid Mini Preparation kit (Tiangen Biotech Beijing 
Co., Beijing, China) and the insert fragment was verified by EcoRI and SalI double restrictive digestion and confirmed by DNA sequencing.

Expression and purification of the recombinant $h H M G B 4$ protein. E.coli BL21 (DE3) was transformed with HMGB4 expression vector $\mathrm{pET} 28 \mathrm{a}(+) / \mathrm{HMGB} 4$ to produce the expression bacterial strain BL21-pET28a(+)/hHMGB4. Next, BL21-pET28a(+)/hHMGB4 cells were induced with $1 \mathrm{mM}$ isopropylthio- $\beta$-D-galactoside (IPTG), harvested and resuspended with TE buffer $(20 \mathrm{mM}$ Tris- $\mathrm{HCl}$ and $1 \mathrm{mM}$ EDTA, $\mathrm{pH}$ 8.0) supplemented with $1 \mathrm{mM}$ phenylmethylsulfonyl fluoride (PMSF). Next, the expression of the recombinant hHMGB4 (rhHMGB4) in the supernatant and the pellets was evaluated by SDS-PAGE followed by Coomassie brilliant blue staining. The procedures are subsequently described in detail.

The pET28a(+)/HMGB4 was transformed into E . coli BL21 (DE3)-competent cells to produce the expression bacterial strain BL21-pET28a(+)/hHMGB4. Small scale testing of antibody expression was performed prior to scaling up the expression procedure. A single colony of E.coli BL21-pET28a(+)/HMGB4 cells was inoculated into $2 \mathrm{ml} \mathrm{LB}$ medium containing $50 \mu \mathrm{g}$ kanamycin $/ \mathrm{ml}$ and incubated at $37^{\circ} \mathrm{C}$ with agitation at $200 \mathrm{rpm}$ overnight in a shaking incubator with a rotational radius of $10 \mathrm{~cm}$. The cells were inoculated into $20 \mathrm{ml}$ fresh LB medium with $50 \mu \mathrm{g}$ kanamycin/ml and grown under the same conditions until the $\mathrm{OD}_{600}$ reached 0.6-1.0. Next, $1 \mathrm{mM}$ of final concentration of IPTG was added for an additional $4 \mathrm{~h}$, the cells were harvested by centrifugation at $10,000 \mathrm{x}$ at $4^{\circ} \mathrm{C}$ for $5 \mathrm{~min}$. Pellet fractions were suspended in $200 \mu \mathrm{l}$ TE buffer with $1 \mathrm{mM}$ PMSF and lysed by sonication on ice at $300 \mathrm{~W}$ for 20 cycles (5 sec on and $10 \mathrm{sec}$ off). The resulting cell lysate was centrifuged at $12,000 \mathrm{x}$ g for $30 \mathrm{~min}$ at $4^{\circ} \mathrm{C}$. The supernatant and pellets were analyzed by $15 \%$ (v/v) SDS-PAGE followed by Coomassie brilliant blue R250 staining.

Inclusion bodies were purified as described elsewhere (9). Briefly, pellets containing inclusion bodies were washed three times with the washing buffer $(50 \mathrm{mM}$ Tris- $\mathrm{HCl}, \mathrm{pH} 8.0,10 \mathrm{mM}$ Triton X-100, 2 M urea and 10 mM EDTA) and resuspended in inclusion body solubilization buffer $(50 \mathrm{mM}$ Tris- $\mathrm{HCl}, \mathrm{pH} 8.0$, $8 \mathrm{M}$ urea, $0.5 \mathrm{M} \mathrm{NaCl}$ and $5 \mathrm{mM}$ imidazole) by stirring for $30 \mathrm{~min}$ at room temperature. The supernatant was collected by centrifugation at $12,000 \mathrm{x} \mathrm{g}$ for $30 \mathrm{~min}$ at $4^{\circ} \mathrm{C}$ and then refolded by urea gradient dialysis (from 8 to $0 \mathrm{M}$ urea). The refolded recombinant protein solution was concentrated and collected to run SDS-PAGE. Finally, the predicted protein band was excised from the gel. rhHMGB4 was retrieved using a Model 422 Electro-Eluter (Bio-Rad, Hercules, CA, USA). The electroelution process was performed at $24 \mathrm{~mA}$ for $2 \mathrm{~h}$ at $4^{\circ} \mathrm{C}$. Finally, the protein concentration was determined by the Bradford assay (10).

Identification of the recombinant $h H M G B 4$ protein by tandem mass spectrometery. The recombinant protein was digested by carboxypeptidase Y (CPY) and the resulting peptide mixture was analyzed by matrix-assisted laser desorption/ionization time-of-flight mass spectrometry (MALDI-TOF)-TOF-MS/MS (4700 Proteomics Analyzer, Applied Biosystems China, Beijing, China). The procedure was performed as follows: Briefly, the rhHMGB4 protein was digested with CPY. Then, the digested sample was admixed with $0.5 \mu \mathrm{l}$ matrix solution containing $5 \mathrm{mg} \mathrm{CHCA} / \mathrm{ml}$. The mixture was vortexed and removed to the sample target, which was allowed to dry in the air at room temperature. The peptide mass fingerprint (PMF) was analyzed in the reflex model and four peptide peaks with the highest ion intensity were measured by tandem mass spectrometery. The MS/MS data were analyzed using De novo Explorer software for de novo sequencing.

Preparation and purification of the anti-hHMGB4 polyclonal antibody. The purified recombinant hHMGB4 protein was emulsified with an equal volume of Freund's complete adjuvant and was injected subcutaneously at six sites in the back of New Zealand rabbits. The study was approved by the Ethics Committee of Shanghai Institute of Planned Parenthood Research. Two weeks later, the rabbits were injected with the hHMGB4 protein with Freund's incomplete adjuvant as a booster immunization. A total of three booster injections were performed at 2 -week intervals. Following the second immunization, serum was separated from the blood collected from the rabbit's ear vein at 2-week intervals to test the antibody titer. The control group was administered with an equal volume of PBS. One week following the final immunization, the antiserum was collected and stored at $-20^{\circ} \mathrm{C}$. The purification of antibodies was performed using affinity chromatography according to the following instructions: The purification of antibodies was performed using affinity chromatography. Serum was mixed with $20 \mathrm{mM}$ Na-phosphate buffer $(\mathrm{pH} 7.0)$ and the mixture was applied to a HiTrap Protein G column (1 ml), equilibrated with $20 \mathrm{mM}$ phosphate buffer ( $\mathrm{pH} 7.0$ ). Following washing of the column with 10 column volume of phosphate buffer, the antibody was eluted by $0.15 \mathrm{M} \mathrm{Gly-HCl}$ (pH 2.5). The resulting elution was collected and neutralized with $1 \mathrm{M}$ Tris- $\mathrm{HCl}$ (pH 9.0) and then stored at $-20^{\circ} \mathrm{C}$.

Identification of the antibody specificity by western blotting. Proteins were separated by $15 \%$ (v/v) SDS-PAGE and transferred onto polyvinylidene fluoride membrane. Following blocking with $5 \%(\mathrm{w} / \mathrm{v})$ non-fat milk, the membrane was incubated with the hHMGB4 polyclonal antibody $(1: 500)$ at $4^{\circ} \mathrm{C}$ overnight, washed with PBST, incubated with HRP-conjugated goat anti-rabbit IgG for $1 \mathrm{~h}$. The bound antibody complexes were detected using SuperSignal ${ }^{\circledR}$ West Pico Chemiluminescent Substrate (Pierce, Rockford, IL, USA) on X-ray films. The preparation of protein samples used is subsequently described: Briefly, the E.coli BL21 cells transformed with the pET28a(+) vector or the recombinant pET28a(+)/HMGB4 plasmid were incubated at $37^{\circ} \mathrm{C}$ to overexpress the recombinant protein. Next, an equal number of bacterial cells were pelleted and lysed for SDS-PAGE and western blotting, as described previously. The proteins were extracted from human prostate cancer cell lines DU145 and LNCaP cells with the RIPA buffer (25 mM Tris-HCl pH 7.6, $150 \mathrm{mM} \mathrm{NaCl,} \mathrm{1 \%} \mathrm{NP-40,}$ $1 \%$ sodium deoxycholate and $0.1 \%$ SDS) added with a cocktail of proteinase inhibitors (Sigma-Aldrich, St. Louis, MO, USA).

Determination of antibody titer by ELISA. Briefly, the purified hHMGB4 protein $(5 \mu \mathrm{g} / \mathrm{ml})$ was coated to a microtiter plate and incubated at $4^{\circ} \mathrm{C}$ overnight. Following blocking with $1 \%$ BSA, 
hHMGB4 antisera with serial dilutions $(1 / 200-1 / 204,800)$ were pipetted into the wells and incubated for $1 \mathrm{~h}$ at $37^{\circ} \mathrm{C}$. Following thorough washing, the HRP-conjugated goat anti-rabbit IgG was added for $1 \mathrm{~h}$ at $37^{\circ} \mathrm{C}$. Color development was performed with the substrate solution containing $3^{\prime}, 3^{\prime}, 5^{\prime}, 5^{\prime}$-tetramethylbenzidine (TMB) and halted by the addition of $1 \mathrm{M} \mathrm{H}_{2} \mathrm{SO}_{4}$. The absorbance at $450 \mathrm{~nm}$ was measured. The antibody titer was defined as the highest antiserum dilution when the P/N value (division of the positive serum $\mathrm{OD}_{450}$ value by the negative control $\mathrm{OD}_{450}$ value) was $>2(\mathrm{P} / \mathrm{N}>2)$.

Immunohistochemical staining. Paraffin-embedded tissue of the normal human prostate and endometrium was purchased from Shanxi Chaoying Co. These tissues were cut into $5 \mu \mathrm{m}$ thick sections and deparaffinized in xylene, then rehydrated with graded ethanol. Antigen retrieval was performed by microwaving the slides using $0.01 \mathrm{M}$ citrate buffers ( $\mathrm{pH}$ 6.0). Endogenous peroxidase activity was removed with $3 \% \mathrm{H}_{2} \mathrm{O}_{2}$ and tissues were blocked with $10 \%(\mathrm{v} / \mathrm{v})$ normal goat serum at $37^{\circ} \mathrm{C}$ for $1 \mathrm{~h}$. The slides were incubated with 1:200 diluted hHMGB4 polyclonal antibodies overnight at $4^{\circ} \mathrm{C}$. Following washing with PBST, slides were incubated with biotinylated goat anti-rabbit secondary antibody, followed by incubation with streptavidin-HRP complex. Color reaction was detected using diaminobenzidine tetrahydrochloride (DAB). Replacement of the hHMGB4 antibody with PBS was conducted as a negative control to confirm specificity. The slides were counterstained with hematoxylin and mounted with neutral resin.

\section{Results}

Construction of the pET28a(+)/hHMGB4 expression plasmid. The 560 bp DNA fragment encoding the full-length hHMGB4 was amplified from plasmid pBluescriptR/hHMGB4 and cloned into the pET28a(+) expression vector between the $E c o$ RI and SalI restriction sites. The recombinant plasmid pET28a(+)/hHMGB4 was confirmed by PCR and restriction enzyme digestion. As shown in Fig. 1, two DNA electrophoretic bands of $\sim 560$ and $\sim 8000$ bp were produced following the EcoRI and SalI restrictive digestion. The target fragment of $\sim 560 \mathrm{bp}$, which was generated following PCR amplification using pET28a $(+) / h H M G B 4$ as the template, is also shown in Fig. 1. DNA sequencing verified that the inserted DNA fragment sequence was as expected (GenBank Accession no. $\mathrm{BC} 021180)$.

Expression and purification of the recombinant $h H M G B 4$. SDS-PAGE analysis revealed that the recombinant hHMGB4 protein was expressed in E.coli BL21 (DE3) cells at an expected band of $28 \mathrm{kDa}$ (Fig. 2). rhHMGB4 was found to be primarily expressed as inclusion bodies composed of insoluble aggregates. More than $80 \%$ soluble recombinant hHMGB4 protein was obtained following extraction with $8 \mathrm{M}$ urea from inclusion bodies and dialyzed (Fig. 2). The purity of the rhHMGB4 reached $>95 \%$ following dialysis and electroelution process.

MS/MS analysis of the recombinant hHMGB4 protein. MALDI-TOF was used to analyze the digested hHMGB4 proteins. Four precursor ion peaks at $\mathrm{m} / \mathrm{z}$ 967.24, 1261.23,

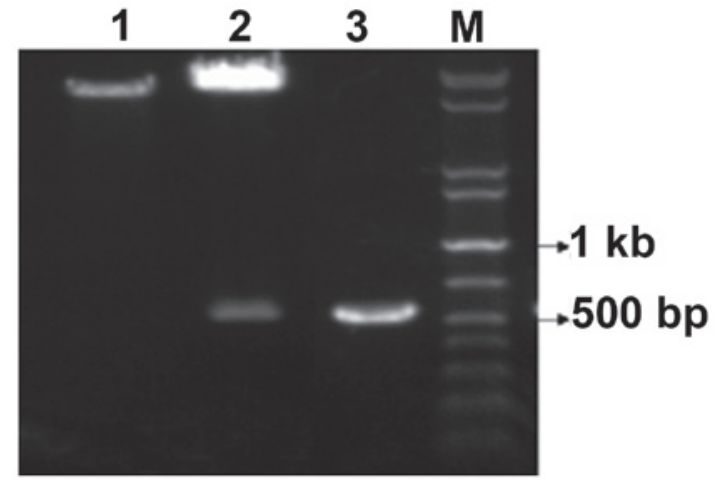

Figure 1. Identification of pET28a(+)/HMGB4 by restriction enzyme digestion and PCR. Lane 1, undigested pET28a(+)/HMGB4; Lane 2, EcoRI and SalI double digestion of pET28a(+)/HMGB4; Lane 3, amplification by PCR of human HMGB4 cDNA and Lane M, 1 kb plus DNA marker.

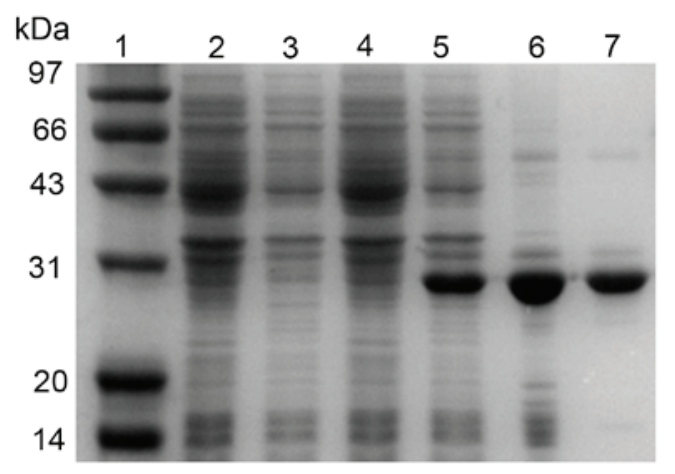

Figure 2. A typical SDS-PAGE profile of rhHMGB4 expressed and purified from $E$. coli BL21. Protein lysates were prepared from E. coli BL21 cells harboring with empty vector (lanes 2 and 3 ) or transformed with pET28a(+)/HMGB4 (lanes 4 and 5). The E. coli were either untreated (lanes 2 and 4) or treated with IPTG (lanes 3 and 5). Protein loaded on lane 6 was prepared from the inclusion bodies. The rhHMGB4 protein purified via electroelution was loaded on lane 7. Lane 1, low molecular weight protein markers. IPTG, isopropylthio- $\beta$-D-galactoside.

1283.21 and 1370.26 were identified, respectively, and PMF analysis suggested that the protein is HMGB4. In addition, 1283.21 was selected for tandem MS analysis. As shown in Fig. 3, the amino acid sequence was exactly matched with the N-terminal sequence of HMGB4 among 29-36 amino acid residues, re-confirming that the purified recombinant protein is rhHMGB4.

Generation of polyclonal antibodies against rhHMGB4 protein. Polyclonal antibodies were generated in rabbits by using the purified rhHMGB4 protein. The rhHMGB4 antigen may be detected with the antisera at the dilution of 1:102,400 by ELISA (as described in Materials and methods). In addition, no immune reactivity was detected with pre-immune serum.

Analysis of the antibody specificity. To analyze the specificity of the prepared HMGB4 polyclonal antibody, the expression of the recombinant hHMGB4 protein was measured using the lysate prepared from the transformed E.coli BL21 cells by western blot analysis. As shown in Fig. 4A, there is a distinguished band of $28 \mathrm{kDa}$ as expected in the 


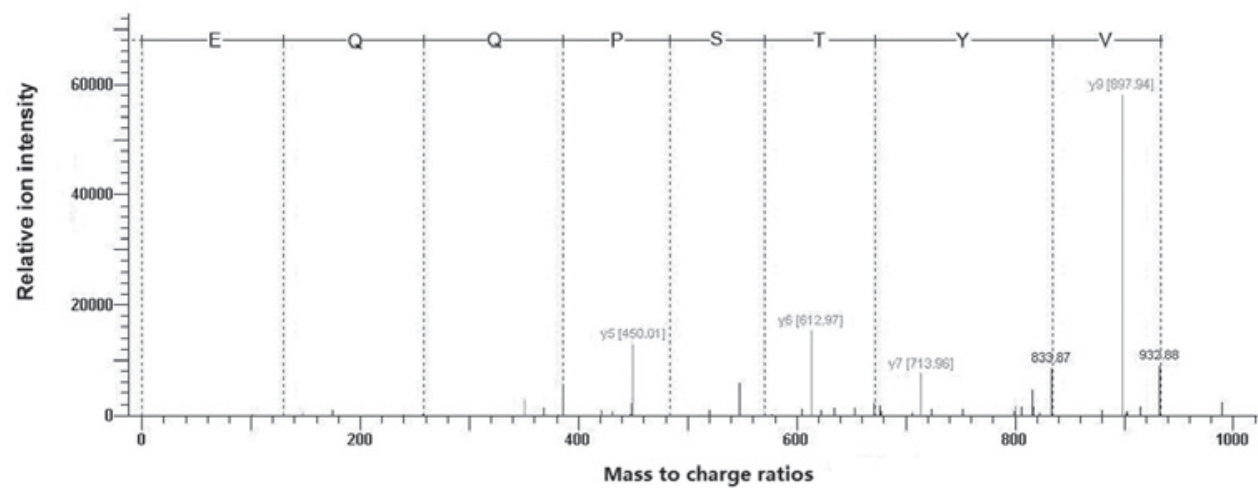

Figure 3. MS/MS spectrum obtained from one hHMGB4 fragment with charged precursor ion at m/z 1283.21 and part amino acid sequence of this peptide. $y$-axis, relative ion intensity; $x$-axis, mass to charge ratios.

A

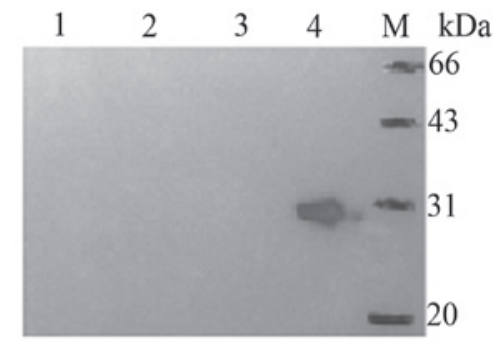

B

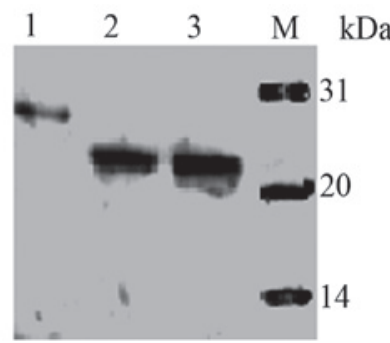

Figure 4. Specificity analysis of the anti-hHMGB4 polyclonal antibody by western blotting technique. (A) Protein lysates were prepared from E. coli BL21 cells harboring with empty vector (lanes 1 and 3) or transformed with pET28a (+)/HMGB4 (lanes 2 and 4). The E. coli were untreated (lanes 1 and 3) or treated with IPTG (lanes 2 and 4). (B) Protein lysates were prepared from human prostate cancer cells DU145 (lane 2) and LNCaP (lane 3). The purified recombinant hHMGB4 protein was used as a positive control (lane 1). IPTG, isopropylthio- $\beta$-D-galactoside.

A

C

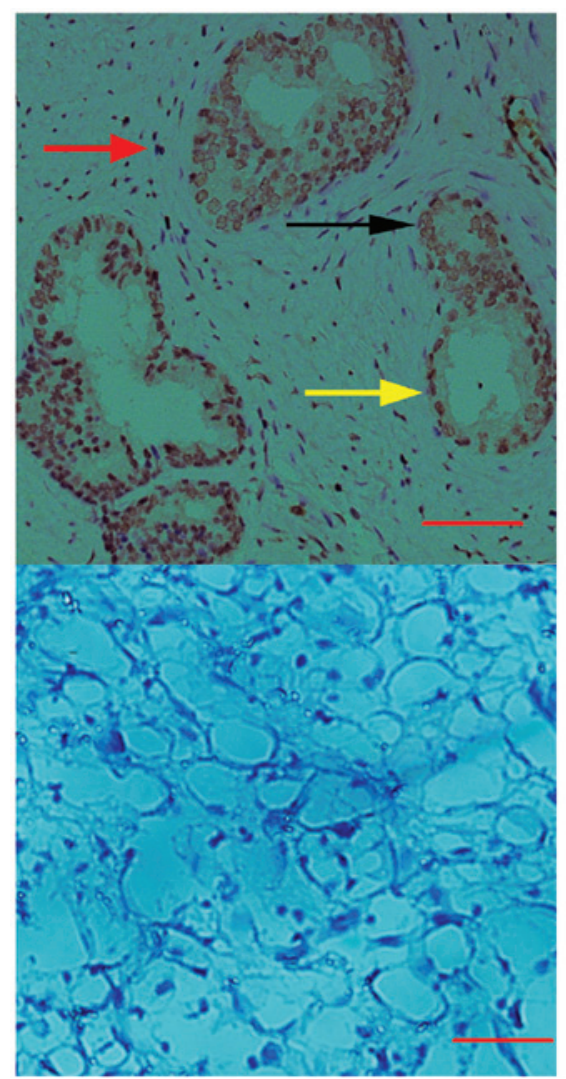

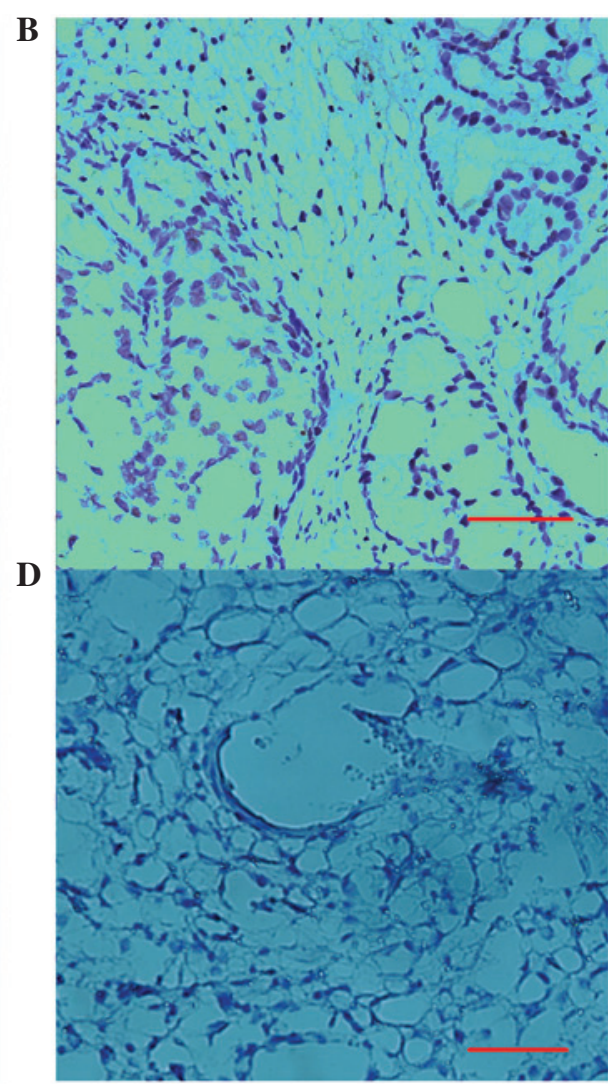

Figure 5. Immunohistochemical analysis of hHMGB4 in human prostate and uterus tissue sections. The slices of (A) human prostate and (C) uterus were stained with the hHMGB4 antibody. Immunostaining of HMGB4 was detected in the nuclei of prostate epithelial cells (black arrow), basal cells (yellow arrow) and stromal cells (red arrow). No significant staining of hHMGB4 was detected in the decidualized endometrium of human uterus. The pre-immunized serum was also used in the immunostaining analysis as the negative control (B and D). Scale bar in A-D, $50 \mu \mathrm{m}$. 
IPTG-treated BL21-pET28a(+)/HMGB4 cells (lane 4), while no band was detected in the BL21-pET28a(+) cells untreated (lane 1) and treated with IPTG (lane 2), as well as the BL21-pET28a(+)/HMGB4 cells treated with IPTG (lane 3).

In addition, hHMGB4 expression was measured in the two human prostate cancer cell lines, DU145 and LNCaP, in the immunoblotting analysis. The results showed that one single highlighted band above $20 \mathrm{kDa}$ was detected in the two cell lines (Fig. 4B), indicating that the antibody recognizes denatured HMGB4 prepared from human cells with a high specificity.

Immunostaining analysis of HMGB4 in human tissue of the prostate and uterus. Immunohistochemical studies were performed in human prostate tissues as well as decidual tissues to further characterize the anti-hHMGB4 antibody. The results in Fig. 5A show that HMGB4 is localized predominantly in the nuclei of epithelial, basal and stromal cells of the prostate, while cytosolic staining in these cells was extremely weak (Fig. 5A). However, no immunostaining of hHMGB4 was detected in the decidual areas of the uterus (Fig. 5C). In addition, no significant staining was observed in the prostate (Fig. 5B) and decidual tissues (Fig. 5D) when the preimmunized serum was used.

\section{Discussion}

The current study reports the successful generation of a polyclonal antibody against the human HMGB4. For production of its antigen, the prokaryotic expression vector pET28a $(+)$ was used to express a fusion protein with the full length human HMGB4 and $\mathrm{His}_{6}$-tags and thrombin at the terminal of the protein. Therefore, the molecular weight of the fusion protein is $\sim 28 \mathrm{kDa}$, detected by SDS-PAGE analysis, higher than the theoretical molecular weight $(22.4 \mathrm{kDa})$ of hHMGB4. In addition, the $28 \mathrm{kDa}$ protein was further validated to be hHMGB4 via MS/MS analysis.

Following rabbit immunization with the purified recombinant hHMGB4, the polyclonal antibody against hHMGB4 was produced with a high titer of antiserum $(1: 102,400)$ determined via ELISA. Notably, only a unique band was detected in the lysate prepared from two prostate cancer cell lines analyzed via western blotting, indicating that the antibody recognized denatured human HMGB4 with a high specificity.

Finally, the antibody was tested for its potential application used in immunohistochemistry. Staining was observed in the prostate but not in uterine. In addition, expression of HMGB4 was measured in 33 human tissues by RT-PCR, showing that expression of HMGB4 is only detectable in the testis and prostate and is absent in others, including uterine (data not shown). The data strongly suggest that the present antibody is a usable reagent in immunostaining analysis.
HMGB1 is not only a nuclear protein, but also promotes prostate cancer progression as a secreted protein (11). However, marked immuniostaining of HMGB4 was observed in the nuclei of epithelial, basal and stromal cells of the prostate, but the staining was weak in the cytoplasm of these cells, suggesting that HMGB4 function is restricted in the nuclei. Future studies are likely to focus on determining whether the HMGB4 antibody is useful in immuno-precipitation assays to perform a functional study of HMGB4 in prostate cancer.

In conclusion, a rabbit polyclonal antibody highly specific for HMGB4 was generated, which may be used for immunostaining and immunoblotting analysis. Availability of this HMGB4 antibody is likely to facilitate the further investigation of human HMGB4.

\section{Acknowledgements}

This study was supported by grants from the National Natural Science Foundation of China (grant no. 81270760), the National Basic Research Program of China (grant no. 2009CB941704) and Shanghai Municipal Committee of Science and Technology (grant nos. 09140903200 and 10ZR1425500).

\section{References}

1. Ueda T and Yoshida M: HMGB proteins and transcriptional regulation. Biochim Biophys Acta 1799: 114-118, 2010.

2. Knapp S, Müller S, Digilio G, et al: The long acidic tail of high mobility group box 1 (HMGB1) protein forms an extended and flexible structure that interacts with specific residues within and between the HMG boxes. Biochemistry 43: 11992-11997, 2004.

3. Stros M: HMGB proteins: interactions with DNA and chromatin. Biochim Biophys Acta 1799: 101-113, 2010.

4. Agresti A and Bianchi ME: HMGB proteins and gene expression. Curr Opin Genet Dev 13: 170-178, 2003.

5. Müller S, Ronfani L and Bianchi ME: Regulated expression and subcellular localization of HMGB1, a chromatin protein with a cytokine function. J Intern Med 255: 332-343, 2004.

6. Ronfani L, Ferraguti M, Croci L, et al: Reduced fertility and spermatogenesis defects in mice lacking chromosomal protein Hmgb2. Development 128: 1265-1273, 2001.

7. Nemeth MJ, Curtis DJ, Kirby MR, et al: Hmgb3: an HMG-box family member expressed in primitive hematopoietic cells that inhibits myeloid and B-cell differentiation. Blood 102: 1298-1306, 2003.

8. Catena R, Escoffier E, Caron C, et al: HMGB4, a novel member of the HMGB family, is preferentially expressed in the mouse testis and localizes to the basal pole of elongating spermatids. Biol Reprod 80: 358-366, 2009.

9. Kou G, Shi S, Wang H, et al: Preparation and characterization of recombinant protein $\mathrm{ScFv}(\mathrm{CD} 11 \mathrm{c})-\mathrm{TRP} 2$ for tumor therapy from inclusion bodies in Escherichia coli. Protein Expr Purif 52: 131-138, 2007.

10. Carlsson N, Borde A, Wölfel S, et al: Quantification of protein concentration by the Bradford method in the presence of pharmaceutical polymers. Anal Biochem 411: 116-121, 2011.

11. Kuniyasu H, Chihara Y, Kondo H, et al: Amphoterin induction in prostatic stromal cells by androgen deprivation is associated with metastatic prostate cancer. Oncol Rep 10: 1863-1868, 2003. 